\title{
BMJ Open Understanding the factors that influence the quality of paid disability support for adults with acquired neurological disability and complex needs: a scoping review protocol
}

\author{
Megan Topping (D) , Jacinta Douglas (D) , ${ }^{1,2}$ Di Winkler (D) 1,2
}

To cite: Topping M, Douglas J, Winkler D. Understanding the factors that influence the quality of paid disability support for adults with acquired neurological disability and complex needs: a scoping review protocol. BMJ Open 2019;9:e034654. doi:10.1136/ bmjopen-2019-034654

- Prepublication history and additional material for this paper are available online. To view these files, please visit the journal online (http://dx.doi. org/10.1136/bmjopen-2019034654).

Received 30 September 2019 Revised 28 November 2019 Accepted 04 December 2019

Q Check for updates

(c) Author(s) (or their employer(s)) 2019. Re-use permitted under CC BY-NC. No commercial re-use. See rights and permissions. Published by BMJ.

${ }^{1}$ Summer Foundation, Blackburn, Victoria, Australia ${ }^{2}$ Living with Disability Research Centre, La Trobe University, Bundoora, Victoria, Australia

Correspondence to Megan Topping; megan.topping@ summerfoundation.org.au

\section{ABSTRACT}

Introduction Adults with disability as the result of an acquired neurological disorder often have varying and complex support needs. Consequently, adults with complex needs often require paid support to enable them to exercise choice and control and participate fully and effectively in society. Given support is so critical for this population, this scoping review aims to further understand the factors that influence the quality of paid disability support for adults with acquired neurological disability and complex needs.

Methods and analysis The methodology proposed by Arksey and 0'Malley, with the modifications recommended by Levac and O'Brien and the Preferred Reporting Items for Systematic Reviews and Meta-Analysis: extension for Scoping Reviews will be used. Systematic and comprehensive electronic database searches will be conducted in MEDLINE, CINAHL, PsycINFO, Scopus and Embase. The review will follow a six-stage framework including (1) identifying the research question/s, (2) identifying the relevant studies, with (3) study selection and (4) charting of data by two independent coders, (5) collating, summarising and reporting data and (6) expert consultation which will be sought from people with lived experience, as well as clinical and academic experts. Work on this scoping review began in June 2019 and will be completed by June 2020 .

Ethics and dissemination Ethical approval will not be required to conduct the scoping review. However, the consultation with people with lived experience will be conducted via codesign workshops. The codesign workshops have received ethical approval from La Trobe University Ethics Committee (reference number: HEC19232). It is intended that review findings will be made available to relevant stakeholders through a peer-reviewed publication, conference presentations and workshops.

\section{INTRODUCTION}

The WHO estimates more than a billion people are living with disability globally, of which approximately 110 million people have very significant difficulties in functioning. ${ }^{1}$ More recently, in 2015, nearly one in five
Strengths and limitations of this study

- This will be the first systematic scoping review to map the factors that influence the quality of paid disability support for adults with acquired disability and complex needs.

- The scoping review will be conducted rigorously in line with Arksey and O'Malley's six-stage systematic approach, incorporating Levac and O'Brien's as well as the PreferredReporting Items for Systematic Reviews and Meta-Analysis: extension for ScopingReviews modifications.

- Ongoing consultation with international and national experts in both clinical and academic settings in this field (DW and JD) and a research librarian (SG) will take place throughout the review process, and people with lived experience of disability and complex needs will be engaged to provide content expertise to inform the interpretation of findings and identify gaps in the literature.

- As this is a scoping review, and not a systematic review, risk of bias assessment will not be conducted.

- Only English language studies will be included in this review.

people in Australia were reported to have a disability, and approximately 1.4 million had a severe or profound core activity limitation, meaning daily support is integral to their quality of life and health outcomes. ${ }^{2}$ Policies both within Australia ${ }^{3}$ and internationally ${ }^{45}$ focus on the rights of adults with disability to participate fully and effectively in society and live as independently as possible. High quality support commensurate with the person's needs and preferences is critical to ensure adults with disability and complex needs can continue to live and participate in the community. The nature and degree of functional impairment experienced by adults with disability, as well as the nature and quality of support provided by paid staff have been 
shown to influence quality of life outcomes for adults with disability. ${ }^{6}$ In addition, the support needs of adults with acquired neurological disability have been found to differ specifically from those of adults with developmental intellectual disability. ${ }^{7}$ Thus this review will focus on adults with disability and complex needs as the result of an acquired neurological disorder for example, from an acquired brain injury, stroke, spinal cord injury or neurological diseases such as multiple sclerosis or Huntington's disease, and adults with cerebral palsy. The decision to focus the review on adults with acquired neurological disability was made to avoid confounding research findings associated with acquired changes in functioning by including research involving adults with developmental intellectual disability whose experiences reflect lifelong functional limitations. Adults with acquired neurological disabilities or cerebral palsy have a range of unique symptoms at varying severities, thus skilled support is necessary to maintain their independent living status. Given the importance of support for this cohort, it is critical to understand the factors that influence the quality of paid disability support.

Support can be a combination of informal (e.g. advocacy or peer support, or support from family and friends) and formal arrangements, ${ }^{8}$ but for people with complex needs paid support is often required. The paid support role is referred to by multiple titles in the literature and in practice including support workers, direct care workers, paid attendant carers or support staff. In this paper, the term disability support worker will be used in a broad sense to refer to all employees who provide direct and daily care to people with disabilities. The disability support worker role is multifaceted and involves a complex range of responsibilities. The primary role of disability support workers is to promote the independence of adults with disability and build their capacity to make their own lifestyle choices, participate in the community and achieve their goals. ${ }^{9}{ }^{10}$ Day-to-day responsibilities include support for daily living and domestic tasks, employment, support to participate in social and leisure pursuits, support with health issues and also to reduce the emotional and physical burden for families. ${ }^{11} 12$ Disability support workers are employed across numerous settings including private residences, family homes, group homes and residential aged care.

Despite the importance of quality support in improving outcomes and enabling people to live with choice and control, ${ }^{13}$ within the past decade the disability workforce in Australia has been criticised for being 'underfunded, unfair, fragmented and inefficient and gives people with a disability little choice and no certainty of access to appropriate supports'. ${ }^{14}$ In recent years however, the provision of disability support services has undergone fundamental reform with a global shift towards individualised funding and a more person-centred approach to care and support. ${ }^{1516}$ In Australia, the National Disability Insurance Scheme (NDIS) was introduced in mid-2013 to implement the federal government's disability policy focusing on increasing the independence and autonomy of people who have "permanent and significant disability'. ${ }^{3}$ This social insurance scheme aims to provide eligible people with disabilities with reasonable and necessary supports to exercise choice and control over their lives and participate in everyday life. ${ }^{17}$ However, in order to achieve this vision, the disability workforce needs to more than double in size by 2019 to $2020^{18}$ and the disability support worker role is becoming increasingly dependent on meeting the needs of a wide range of individuals, meaning specialised skills and knowledge are required. ${ }^{19} 20$

Although the principles of a person-centred approach to disability support are promising, there are concerns about the capacity of the disability workforce in implementing the principles in practice. Since the roll out of the NDIS, the disability support worker role has had the largest increase in casualisation, the largest number of vacancies and the biggest reduction in experience levels in the disability sector. ${ }^{21}$ Providers are hiring individuals without appropriate qualifications or experience at a lower rate for casual or contract positions..$^{21} 22$ This in turn increases staff turnover rate and reduces the quality of support for adults with disability and complex needs. The privatisation of support services also means inexperienced, unqualified individuals can present themselves as disability support workers without background checks, ${ }^{13}$ and formal performance modelling across disability services is limited. ${ }^{23} 24$ Although adults with disability in theory have more choice and control under the new arrangement, without sufficient information to make an informed decision, adults with disability may choose support workers based on their relatability, for example, similar interests and age, rather than their capacity, qualifications or experience. ${ }^{25}$ While there is evidence that a better relationship between client and support worker can facilitate better support, ${ }^{12}{ }^{26}$ this does not necessarily result in quality support. ${ }^{27}$ In order to empower adults with disability to truly exercise choice and control and make informed decisions as they navigate support systems, we first need to understand the factors that influence the quality of paid disability support.

Considering the above, this scoping review aims to (1) examine and map the factors that influence the quality of paid disability support for people with acquired neurological disability and complex needs from the perspective of people with lived experience, close others of people with lived experience and the disability workforce; (2) summarise and disseminate research findings to inform policymakers, practitioners and consumers and (3) identify gaps in the existing literature by drawing conclusions regarding the current state of the research activity.

\section{METHODS AND ANALYSIS}

The systematic scoping review method chosen to conduct this scoping review has been informed by the rigorous approach proposed by Arksey and O'Malley ${ }^{28}$ with the methodology modifications recommended by Levac 
and $\mathrm{O}^{\prime} \mathrm{Brien}^{29}$ as well as the Preferred Reporting Items for Systematic Reviews and Meta-Analysis: extension for ScopingReviews (PRISMA-ScR). ${ }^{30}$ Scoping review methodology $^{1231}$ is primarily useful for mapping the current state of the literature and identifying knowledge gaps around a broad and complex topic ${ }^{32}$ consistent with the aim of the current review. This review will follow the six steps outlined by Arksey and O'Malley ${ }^{28}$ : identifying the research question; identifying relevant studies; study selection; charting the data; and collating, summarising and reporting the results, including implications for policy, practice or research and expert consultation. Work on this scoping review began in June 2019 and will be completed by June 2020 .

\section{Identifying the research question}

The central research question for this review is: What are the factors that influence the quality of paid disability support for people with acquired neurological disability and complex needs?

\section{Identifying relevant studies (developing the search)}

The search strategy was developed by the authors in consultation with content experts and a research librarian (see online supplementary file for the full MEDLINE search). Preliminary searches of the MEDLINE and Embase databases helped refine the search terms and strategy. Initially, three very broad keyword terms were searched: 'disability', 'support' and 'quality' combined with the Boolean operator 'AND'. This search yielded an unmanageable number of articles, including many irrelevant articles, but provided keywords and further search terms to include. Using this initial search, prior knowledge of the topic area, and known peer-reviewed literature, a comprehensive list of search terms was developed. The search terms were iteratively tested and refined on MEDLINE by monitoring the yield after including or eliminating any term and checking the first 100 references for relevance. Subject heading and Medical Subject Headings $(\mathrm{MeSH})$ term tree structures were checked to assess whether broader or narrower terms should be included. The initial search strategy included three concept headings: (1) acquired disability, (2) paid support and (3) barriers and facilitators. Terms within the concepts were combined using the operator OR and the three concepts were combined using the operator AND. After preliminary searches on MEDLINE with the final search terms, the third concept, barriers and facilitators, was removed as the search became too limited and missed key relevant articles. Thus, the search will combine the two broad concepts: (1) acquired disability (population) and (2) paid support (concept) to identify relevant literature.

The following five electronic databases will be searched: MEDLINE, CINAHL, PsycINFO, Scopus and Embase. $\mathrm{MeSH}$ terms will be added in MEDLINE, CINAHL and PsycInfo where appropriate, and equivalent Emtree headings will be added in Embase. The search terms will be adapted for use with each bibliographic database. The search will be limited to studies only involving human participants published in English since 2009. The reference lists of eligible studies and any review articles that we identify will also be checked for relevant papers. The search strategy will be registered in each database to ensure we receive new, eligible studies once they appear online after the search date.

\section{Study selection}

Eligibility criteria

Peer-reviewed articles with extractable primary research data will be eligible for inclusion. All study designs will be considered including qualitative and quantitative methods. These include cross-sectional, between subjects, within subjects, longitudinal, randomised controlled trials, case-control, observational studies, multimethod studies, pilot studies and trend studies, book chapters, commentary/opinion articles, study protocols, editorials and conference proceedings. Conference proceedings will be excluded as we only seek to include peer-reviewed texts and the peer-review process for proceedings is not always explicit.

Only papers reported in English language involving human participants, published since 2009 will be included. The reason for including studies since 2009 is due to the significant changes in the disability system in the past decade. With the international trend towards individualised funding and budgets, the needs and preferences of adults with disability have become core determinants of the demands on the paid support workforce. ${ }^{33} 34$ A more contemporary understanding of the factors that influence the quality of paid disability support will have greater potential to influence current policy and practice.

While the included articles must provide empirical data on the factors that influence the quality of support for people with an acquired neurological disability, the participants in the research could be people with lived experience, close others/informal supports or the disability workforce. The perspective of the data will be considered during analysis.

With regards to the content of the papers, we expect following initial screening the eligibility criteria will be tightened, in line with Levac and O'Brien's ${ }^{29}$ methodology. The a priori inclusion criteria are listed below.

\section{Inclusion criteria}

1. Clinical population: Young people (18 to 65 ) with disability and complex needs as the result of an acquired neurological disorder for example, from an acquired brain injury, stroke, spinal cord injury or neurological diseases such as multiple sclerosis or Huntington's disease, and adults with cerebral palsy.

2. Research population: The specified clinical population above, close others or the disability workforce.

3. Concept: Studies that report on the factors that influence the quality of paid disability support for adults with acquired disability and complex needs. 
4. Peer-reviewed articles with extractable primary research data. All study designs will be considered including qualitative and quantitative methods. These include cross-sectional, between subjects, within subjects, longitudinal, randomised controlled trials, casecontrol, observational studies, multimethod studies, pilot studies and trend studies.

5. English language studies only involving human participants published since 2009.

\section{Screening}

The results of the literature searches will be exported and managed in Covidence, EndNote and Excel. Duplicate references will be removed. All of the identified citations will be independently assessed by at least two of the four independent reviewers using a predefined relevance criteria form, with the principal reviewer reviewing all citations. The reviewers will discuss the screening process and highlight any uncertainties related to study selection, in order to refine the search strategy if needed. Agreement between the two reviewers will be assessed. If agreement exceeds $80 \%$, full-texts will be retrieved whenever at least one reviewer included the citation. If the agreement is less than $80 \%$, screening criteria will be discussed further and tightened, if necessary, to ensure that the reviewers have consistent interpretations of the screening criteria. The reviewers will then overlap on a larger proportion of titles until agreement is satisfactory $(>80 \%)$. Conflicts will be discussed if agreement remains $<80 \%$ after all citations are screened by at least two reviewers.

The full-text articles will be retrieved for all references included after the title and abstract screening. The principal reviewer will read all articles retrieved for full-text evaluation, and two of the other research team members will independently read half of the literature each, so that all articles have been reviewed by two reviewers. Any differences of opinion will be discussed and the eligibility criteria will be tightened further if necessary. If agreement cannot be reached discrepancies will be resolved by consultation with the third reviewer. If agreement still cannot be reached, the paper will be included.

The reference lists of studies that are included based on review of the full-text article will be hand searched to find any further relevant citations. The process of study selection and the number of studies at each stage of the selection process will be reported using the PRISMA flow chart. $^{35}$

\section{Charting the data (data extraction)}

The review team will iteratively refine the data extraction form through the screening process when refining the eligibility criteria, in order to answer the research question appropriately. It is anticipated that for each of the included studies, the following data will be extracted: (1) study characteristics: year study conducted, study design and methodology and measures, (2) participant characteristics: population type (people with disability, close others, informal support, disability workforce), demographics, disability-related characteristics, support type and amount, living situation, (3) author's description of paid support, for example, disability support worker, personal care assistant, (4) findings relating to the factors that influence the quality of paid disability support, (5) identified gaps in the literature and research implications. Tables will be purposively built by the review team iteratively during the screening process once the nature of the data is known. Two reviewers will independently extract data from 5 to 10 studies, depending on the number of eligible full-text articles, to determine the consistency of the data extraction approach between reviewers.

Given that the aim of the scoping review is to explore the breadth, as opposed to depth, of the available evidence addressing the research question, and inclusively reflect the perspectives of people with acquired neurological disability and complex needs, close others, informal supports and the disability workforce, a critical appraisal will not be conducted. This will enable us to identify the nature and extent of the evidence around factors that influence the quality of support for people with acquired neurological disability and complex needs, regardless of the research aims and methodologies of included studies.

\section{Collating, summarising and reporting the results}

The PRISMA-ScR checklist for scoping reviews will be used to guide the collating, summarising and reporting of results. ${ }^{30}$ A descriptive numerical summary of the scoping review including the numbers of sources screened, included and excluded at each stage, will be presented in a PRISMA flow diagram.

A summary of the key characteristics of the eligible articles, including the study and participant characteristics, the perspective of the data and the paid support descriptions, will be presented in tables, as appropriate. A high-level summary of the key characteristics will be provided in text. Given the nature of the literature, it is anticipated that the majority of the research will be qualitative. However, where eligible, quantitative studies will be included. Qualitative and quantitative data will be examined separately. The data relevant to the research question, on the factors that influence the quality of paid disability support, will be presented for each individual source of evidence and sorted by perspective of the data (eg, people with disability, close others, informal supports or the disability workforce). A narrative synthesis will be conducted to summarise the findings related to the research question based on themes that emerge from the extracted data. Subsequently, a list of unique factors will be organised into the themes and presented descriptively in table format. While the primary goal is to present the factors that shape quality paid support, additional data relating to factors that facilitate a positive working relationship between the support worker and client, as well as any data relating to what makes an excellent support worker, will be presented. Finally, any gaps in the literature identified by authors of included texts will be 
extracted and considered against the gaps we observe from the scoping review findings.

\section{Patient and public involvement}

People with lived experience of disability and complex needs will be invited to participate in a codesign workshop to explore 'what makes an excellent support worker?' and discuss preliminary findings of the scoping review. People with disability will be recruited via Summer Foundation and La Trobe University's Living with Disability Research Centre's existing networks. The workshops will be conducted at an accessible location local to the participants and travel costs will be covered. The finalised structure and scope of the workshop will be informed by the preliminary findings of the review, but we anticipate two workshops will be conducted, each with up to six participants. We plan to conduct each of the workshops in two phases. During phase 1, participants will be invited to share their perspective on the factors that influence the quality of support and what they feel are barriers to quality support. Phase 2 will involve sharing the preliminary findings of the scoping review with the workshop participants. The scoping review findings will be prepared and presented in an accessible format. After time to review the findings and discuss in smaller groups, facilitated by the researchers, participants will be invited to provide feedback on the findings with reference to the factors they highlighted in phase 1. Additionally, to gauge the importance of different factors, we may ask participants to rank the identified factors or choose their top three most important factors. Finally, workshop participants will be asked to share their thoughts and recommendations around methods of dissemination and knowledge translation of the scoping review findings. This consultation process will help inform the interpretation and dissemination of the findings and identify further gaps in the literature.

\section{ETHICS AND DISSEMINATION}

The scoping review does not require ethical approval. However, the consultation with people with lived experience will be conducted via codesign workshops. The codesign workshops have received ethical approval from La Trobe University Ethics Committee (reference number: HEC19232) as part of a larger project to improve disability supports for people with cognitive and communication impairments.

The aim of this scoping review is to produce a synthesis of the factors that influence the quality of paid disability support for adults with acquired disability and complex needs, based on reviewing the relevant literature. The proposed review has the potential to impact practice and policy by improving clarification around how to facilitate high quality, effective support for adults with acquired disability and complex needs. We also hope the review will expand the knowledge of people who are accessing and choosing support services and provide a better understanding of what factors facilitate effective support from disability support workers, and what the barriers to receiving quality support are. It is intended that review findings will be made available to relevant stakeholders through a peer-reviewed publication, conference presentations and workshops.

Acknowledgements The authors gratefully acknowledge Dr Sue Gilbert (SG) for assistance with the protocol.

Contributors All authors have made a substantial intellectual contribution. MT lead the conceptualisation of the review. MT, JD and DW contributed to the scope and design of the review and developed the search strategy. All authors contributed to editing and revising the manuscript and gave approval for publication of this protocol.

Funding This work was funded by Summer Foundation in partnership with La Trobe University.

Competing interests None declared.

Patient consent for publication Not required.

Provenance and peer review Not commissioned; externally peer reviewed.

Open access This is an open access article distributed in accordance with the Creative Commons Attribution Non Commercial (CC BY-NC 4.0) license, which permits others to distribute, remix, adapt, build upon this work non-commercially, and license their derivative works on different terms, provided the original work is properly cited, appropriate credit is given, any changes made indicated, and the use is non-commercial. See: http://creativecommons.org/licenses/by-nc/4.0/.

\section{ORCID iDs}

Megan Topping http://orcid.org/0000-0001-6008-7943

Jacinta Douglas https://orcid.org/0000-0003-0940-6624

Di Winkler https://orcid.org/0000-0003-3899-6248

\section{REFERENCES}

1 WHO. World report on disability, 2011. Available: https://www.who. int/disabilities/world_report/2011/en/ [Accessed June 2019].

2 Aihw. Australia's welfare 2017 - Full Report, 2017. Available: https://www.aihw.gov.au/getmedia/088848dc-906d-4a8b-aa0979dfOf943984/aihw-aus-214-aw17.pdf.aspx?inline=true [Accessed June 2019].

3 Cukalevski E. Supporting Choice and Control-An Analysis of the Approach Taken to Legal Capacity in Australia's National Disability Insurance Scheme. Laws 2019;8.

4 United Nations General Assembly. Convention on the rights of persons with disabilities: Resolution/adopted by the general assembly (A/RES/61/106), 2007. Available: http://www.unhcr.org/ refworld/docid/45f973632.html [Accessed June 2019].

5 United Nations General Assembly. Report of the special Rapporteur on the rights of persons with disabilities (theme: access to RightsBased support for persons with disabilities) (A/HRC/34/58), 2016. Available: https://www.ohchr.org/EN/Issues/Disability/SRDisabilities/ Pages/Reports.aspx [Accessed June 2019].

6 Bigby C, Beadle-Brown J. Improving quality of life outcomes in supported accommodation for people with intellectual disability: what makes a difference? J Appl Res Intellect Disabil 2018;31:e182-200.

7 Bigby C, Douglas J, Bould E. Developing and maintaining person centred active support: a demonstration project in supported accommodation for people with neurotrauma, 2018. Available: http:// hdl.handle.net/1959.9/563228 [Accessed November 2019].

8 United Nations Committee on the Rights of Persons with Disabilities. General Comment No. 1. article 12: equal recognition before the law. Available: https://www.ohchr.org/en/hrbodies/crpd/pages/gc.aspx [Accessed June 2019].

9 Fisher KR, Gendera S, Graham A, et al. Disability and support relationships: what role does policy play? Aust J Publ Admin 2018;9:37-55.

10 Australian Government Department of Social Services. NDIS quality and Safeguarding framework, 2016. Available: https://www.dss. gov.au/sites/default/files/documents/04_2017/ndis_quality_and_ safeguarding_framework_final.pdf [Accessed June 2019]. 
11 Clarke J. Consumers, clients or citizens? politics, policy and practice in the reform of social care. European Societies 2006;8:423-42.

12 Miller EL, Opie ND. Severely disabled adults and personal care attendants: a pilot study. Rehabil Nurs 1987;12:185-7.

13 Jorgensen D, Parsons M, Reid MG, et al. The providers' profile of the disability support workforce in New Zealand. Health Soc Care Community 2009;17:396-405.

14 Productivity Commission. Disability care and support: productivity Commission inquiry report overview and recommendations, 2011. Available: www.pc.gov.au [Accessed June 2019].

15 Da Roit B, Le Bihan B. Similar and yet so different: cash-for-care in six European countries' long-term care policies. Milbank $Q$ 2010;88:286-309.

16 Green J, Mears J. The implementation of the NDIS: who wins, who loses? Cosmopolitan Civil Societies: An Interdisciplinary Journal 2014;6:25-39.

17 National Disability Insurance Agency. Annual report 2015-16, 2016. Available: https://www.ndis.gov.au/about-us/publications/annualreport/annual-report-2015-16 [Accessed November 2019].

18 Australian Government Productivity Commission. National disability insurance scheme (NDIS) costs: productivity Commission study report, 2017. Available: https://www.pc.gov.au/inquiries/ completed/ndis-costs/report/ndis-costs-overview.pdf [Accessed June 2019].

19 Hewitt A, Larson S. The direct support workforce in community supports to individuals with developmental disabilities: issues, implications, and promising practices. Ment Retard Dev Disabil Res Rev 2007;13:178-87.

20 National Disability Services. State of the disability sector report, 2017. Available: https://www.nds.org.au/images/news/State_of_the Disability_Sector_report_2017.pdf [Accessed June 2019].

21 Mavromaras K, Moskos M, Mahuteau S. Evaluation of the NDIS. final report. Adelaide National Institute of Labour Studies, Flinders University; 2018.

22 Baker B. The Hunt for Disability: The New Eugenics and the Normalization of School Children. In: Allan J, Artiles AJ, eds. World Yearbook of education. Routledge, 2017: 137-73.
23 National Disability Services. Australian disability workforce report: 3rd edition July 2018. Available: https://www.nds.org.au/images/ workforce/ADWR-third-edition_2018_final.pdf [Accessed June 2019].

24 Lawn S, Westwood T, Jordans S, et al. Support workers can develop the skills to work with complexity in community aged care: an Australian study of training provided across aged care community services. Gerontol Geriatr Educ 2017;38:453-70.

25 Harry ML, MacDonald L, McLuckie A, et al. Long-Term experiences in cash and counseling for young adults with intellectual disabilities: familial programme representative descriptions. J Appl Res Intellect Disabil 2017;30:573-83.

26 McCluskey A. Paid attendant carers hold important and unexpected roles which contribute to the lives of people with brain injury. Brain Inj 2000;14:943-57.

27 Opie ND, Miller ET. Attribution for successful relationships between severely disabled adults and personal care attendants. Rehabil Nurs 1989;14:196-9.

28 Arksey H, O'Malley L. Scoping studies: towards a methodological framework. Int J Soc Res Methodol 2005;8:19-32.

29 Levac D, Colquhoun H, O'Brien KK, O’Brien K. Scoping studies: advancing the methodology. Implementation Sci 2010;5.

30 Tricco AC, Lillie E, Zarin W, et al. PRISMA extension for scoping reviews (PRISMA-ScR): checklist and explanation. Ann Intern Med 2018;169:467-73.

31 Colquhoun HL, Levac D, O'Brien KK, et al. Scoping reviews: time for clarity in definition, methods, and reporting. J Clin Epidemiol 2014;67:1291-4.

32 Davis K, Drey N, Gould D. What are scoping studies? A review of the nursing literature. Int J Nurs Stud 2009;46:1386-400.

33 Burtt S. Disability policy: are we making progress? Soc Phil Pol 2017;34:259-76.

34 Harry ML, Mahoney KJ, Mahoney EK, et al. The cash and counseling model of self-directed long-term care: effectiveness with young adults with disabilities. Disabil Health J 2017;10:492-501.

35 Liberati A, Altman DG, Tetzlaff J, et al. The PRISMA statement for reporting systematic reviews and meta-analyses of studies that evaluate health care interventions: explanation and elaboration. PLoS Med 2009;6:e1000100. 